and an understanding of withdrawal and its natural history is rarely considered from the user's perspective.

Aims/Methods: This presentation will outline the results of a study of 150 dependent psychostimulants users and their experiences of withdrawal treatment and of withdrawal. A detailed retrospective natural history was documented using a structured and semistructured interview format.

\section{Identification of a bipolar disorder susceptibility locus on chromosome 150}

\author{
E McAuley ${ }^{1,2,3}$, I Blairi, ${ }^{2,3}$, J Fullerton ${ }^{1,2,3}$, J Donald ${ }^{4}$, \\ P Mitchel| ${ }^{3,5}$, P Schofield ${ }^{1,2,3}$ \\ 'Prince of Wales Medical Research Institute; ${ }^{2}$ Garvan Institute; ${ }^{3} \mathrm{UNSW}$; \\ ${ }^{4}$ Deptartment of Biological Sciences, Macquarie University; and ${ }^{5}$ The Black Dog \\ Institute, Prince of Wales Hospital, Sydney, Australia
}

Background: Bipolar affective disorder (BP) is a relatively common, severe mood disorder characterized by periods of mania and depression, with estimates of lifetime prevalence up to $4 \%$.

Method: We conducted a 10-cM genome scan on 35 multigenerational pedigrees with 288 genotyped individuals (130 affected according to a broad disease definition). Subsequent fine mapping was conducted on the region with significant linkage results and was assessed using parametric, nonparametric and multipoint linkage analysis methods, as well as haplotype analysis based on pedigree-specific, identical-bydescent allele sharing.

Results: The genome scan identified significant linkage on chromosome 15q25-26 and suggestive evidence on chromosomes 4q, 6q and 13q. Analysis of the 15q25-26 region, including additionally typed chromosome $15 \mathrm{q}$ markers, gave significant results with a maximum two-point LOD score of 3.38 and a multipoint LOD score of 4.58 for marker D15S130. A maximum NPL score of $3.38(P=0.0008)$ was obtained at $107.16 \mathrm{cM}$ near D15S130. The $95 \%$ confidence interval estimation suggested a support interval spanning $17 \mathrm{cM}$ between the markers D15S979 and D15S816. Haplotype analysis supported the $95 \%$ confidence interval estimates.

Conclusions: The significant and supporting results from a number of analysis methods performed on chromosome $15 q 25-26$ provide evidence for a BP susceptibility locus in this region. It is further supported by linkage findings from studies on recurrent early-onset major depressive disorder, BP with psychotic features, and a study of schizophrenic and BP subjects, suggesting that the locus might contain a gene conferring susceptibility to both mood and psychotic disorders.

\section{A blinded, placebo-controlled randomized trial of low-dose risperidone, intensive psychological treatment and befriending in young people at risk of psychotic disorder: baseline characteristics of the sample}

\author{
P McGorry', A Yung', S Francey', L Phillips ${ }^{1,2}$, \\ B Nelson'
}

'ORYGEN Research Centre; and '2Department of Psychology, The University of Melbourne and ORC, Melbourne, Australia

Background: Intervention during the prodromal phase of psychotic disorder has become an important focus of early intervention research.

Method: The PACE Clinic, ORYGEN Youth Health, has been conducting a blinded randomized controlled trial (RCT) comparing the effectiveness of low-dose risperidone $(0.5-2.0 \mathrm{mg} /$ day $)$ and intensive CBTbased psychological treatment vs. placebo and intensive CBT-based psychological treatment vs. placebo and a control psychological treatment (befriending). The trial consists of a 12-month treatment phase, followed by a 12-month follow-up phase. The primary outcome of interest is the proportion of patients meeting onset of psychosis criteria during the treatment and follow-up phases.

Results: The current presentation will describe baseline characteristics of the sample. About 119 participants (mean age $=18.36$ years, men $=41.2 \%$ ) meeting ultrahigh-risk criteria for psychotic disorder were randomized to the three treatment groups. Baseline characteristics will be compared between 1) the three treatment groups and 2) the treatment groups and a monitoring group $(n=83$, mean age $=18.45$ years, men $=41 \%$ ), who received 'treatment as usual'.

Conclusion: Pending.

\section{The defensive function of persecutory delusions: an investigation using the Implicit Association Test}

\author{
R McKay ${ }^{1}$, R Langdon², M Coltheart ${ }^{2}$ \\ 'Charles Sturt University; and ${ }^{2}$ Macquarie Centre for Cognitive Science, \\ Macquarie University, Sydney, Australia
}

Background: Delusions are first-rank symptoms of schizophrenia. Of all delusional themes, delusions of persecution are the most commonly observed clinically and the most vigorously researched empirically. Bentall et al. claim that persecutory delusions are constructed defensively, for the maintenance of selfesteem. A central prediction of their model is that 
paranoid individuals will show normal or high selfesteem on overt measures, whereas covert measures will show hidden feelings of low self-esteem.

Methods: The present study used a new methodology that has been widely used in investigations of implicit attitudes, the Implicit Association Test (IAT), to assess covert self-esteem and to test the above prediction. Overt self-esteem was assessed using the Rosenberg Self-Esteem Scale and an adjective self-relevance ratings measure. These measures were administered to 10 patients with acute persecutory delusions, 10 patients with persecutory delusions in remission and 19 matched healthy control participants.

Results: Patients with acute persecutory delusions were found to have lower covert self-esteem (as assessed using the IAT) than healthy controls and patients with remitted persecutory delusions. On the two measures of overt self-esteem, however, the persecutory deluded group did not differ significantly from the other groups once the effects of comorbid depression had been taken into account.

Conclusions: The results of the present study are consistent with a model of persecutory delusions as serving a defensive function. As such, they are consistent with a psychotherapeutic approach to what are perhaps the most frequently observed symptoms of schizophrenia.

\section{Impaired psychosocial functioning in bipolar disorder: the relative contributions of inter-episodic symptoms and illness episodes}

\section{P Mitchell'1, , G Malhi' ${ }^{2,3,4}$, J Ball', A Johnston', D Hadzi-Pavlovic' ${ }^{1}$, J Corry ${ }^{1}$}

'School of Psychiatry, University of New South Wales; ${ }^{2}$ The Black Dog Institute; ${ }^{3}$ Academic Discipline of Psychological Medicine, Northern Clinical School, The University of Sydney; and "Prince of Wales Medical Research Institute, Sydney, Australia

Background: This study examines the relationship between the presence of inter-episodic symptomatology, the frequency of depressed and (hypo)manic episodes, and impairment across eight different domains of functioning. Method: Patients $(n=217)$ attending a tertiary referral center for the assessment of bipolar disorder, who met DSM-IV criteria for BP I or BP II disorder, were included in the analysis. Data were collected on 10 common inter-episodic symptoms reflecting both mood and anxiety, and the frequency of depressive and (hypo)manic episodes. Impairment in the following functional domains was also determined: occupation, financial affairs, self-care, housekeeping, intimate and nonintimate relationships, and parenting. Logistic regression analyses were conducted to examine the number of inter-episodic symptoms reported and the frequency of frank bipolar disorder episodes as predictors of impairment in eight domains of psychosocial functioning.

Results: Psychosocial functional impairment and inter-episodic symptoms were highly prevalent in the sample. The number of inter-episodic symptoms was significantly associated with relationship impairment and employment status, while the frequency of (hypo)manic episodes was associated with impairment in housekeeping and parenting roles. There was no apparent relationship between frequency of depressive episodes and functional capacity.

Conclusions: Both the 'dosage' of inter-episodic (subsyndromal) symptoms and the frequency of (hypo)manic episodes appear to be significant and independent determinants of psychosocial functioning. It is possible that certain aspects of bipolar disorder have a greater impact on some functional domains. Possible methodological reasons for the failure to show a relationship with the frequency of depressive episodes are discussed.

\section{A factor analytic study in bipolar depression, and response to lamotrigine}

\author{
P Mitchell ${ }^{12}$, G Malhi' ${ }^{2,3,4}$, D Hadzi-Pavlovic ${ }^{1}$ \\ 'School of Psychiatry, University of New South Wales; ${ }^{2}$ The Black Dog Institute; \\ ${ }^{3}$ Academic Discipline of Psychological Medicine, Northern Clinical School, \\ The University of Sydney; and APrince of Wales Medical Research Institute, \\ Sydney, Australia
}

Background: This study aimed to identify and compare factors of a 31-item version of the HDRS (HDRS-31) in large samples of patients with bipolar and unipolar depression, then examine for any responsiveness of such factors to the anticonvulsant agent lamotrigine in the bipolar depressed sample.

Methods: This multivariate analytical study was performed on two large depressed samples (one bipolar and the other unipolar) that had been recruited for separate double-blind placebo-controlled trials of lamotrigine. Both studies had very similar designs and assessment tools, the major measures being the MADRS and HDRS-31. To identify the constructs underlying the scale, exploratory factor analyses were applied to the HDRS-31. Treatment responsiveness in the bipolar depressed sample - as indicated by improvement in the total MADRS and HDRS-31, as well as any HDRS factors - was examined using both a mixed-effects analysis and individual time-point $t$-tests.

Results: Seven factors of the HDRS-31 were identified: I - 'depressive cognitions', II - 'psychomotor retardation', III - 'insomnia', IV - 'hypersomnia', 